\title{
An Innovative Cognitive Architecture for Humanoid Robot
}

\author{
Muhammad Faheem Mushtaq ${ }^{1,2}$, Urooj Akram², Adeel Tariq ${ }^{2}$, Irfan Khan ${ }^{1,2}$, Muhammad Zulqarnain ${ }^{2}$, Umer Iqbal ${ }^{2}$ \\ ${ }^{1}$ Department of Computer Science and Information Technology, \\ The Islamia University of Bahawalpur, \\ Bahawalpur, Pakistan \\ ${ }^{2}$ Faculty of Computer Science and Information Technology, \\ Universiti Tun Hussein Onn Malaysia (UTHM), \\ Johor, Malaysia
}

\begin{abstract}
Humanoid robot is appearing as most popular research tool and emerging research field. The greatest challenge in the development of robot is cognition, advancement and the understanding in the human like cognition. Humanoid robot requires a self-learning behavior like the humans that is able to get the experience from environment. Based on experience, it can modify their actions, or having conscious intellectual capability to reduce empirical factual knowledge. In this regard, we propose a novel framework called an Innovative Cognitive Architecture for Humanoid Robot (ICAHR) that is capable to develop cognitive through social interaction and autonomous exploration. It combines the modules of active memory, decision processor, and sensor listener that has capability to perform selflearning behavior like human, to make decisions in dynamic environment, and perform more valid and intelligent actions with better precision. The proposed architecture may result in safe, robust, flexible, and reliable machines that can be substitute of human beings in different tasks. The feasibility of new proposed ICAHR design has been examined through real-world case studies.
\end{abstract}

Keywords-Humanoid robots; cognition; cognitive architecture; self-learning behavior; dynamic environment

\section{INTRODUCTION}

Cognitive is the mental process of knowing that includes the characteristics such as perception, judgment, reasoning, self-examining, decision making, awareness, imagination, memory and emotion. Cognition process or mental process terms are used for all those actions that human being can perform with their mind. The Greek philosopher highlights the importance of cognitive field which is based on experimental proof and accurate information that are collected through complete observation and careful experimentation [1], [2]. The development of machines like human beings required to combine many useful and desirable features including real human like movement, cognition, and human-friendly behavior and design. Humanoid robotics is a challenging and emergent research area. It brings dominant attention in various models about the description of mind and its related phenomena, and play a vital role in $21^{\text {st }}$ century in the field of robotics. The engagement of humanoid robots in some familiar problems likes the processing of information same as human brain and to deal with the real world to perform more tasks with better precision. The physical appearance and locomotion has resemblance like the human in their reasoning, actions, and communication about the real environment [3], [4]. The development of such kind of robot need vast range of discipline in their integration and coordination research efforts in several fields such as human machine interaction, control theory, artificial intelligence, computational and psychological computational and perception neuroscience [5]-[10].

Humanoid robots are especially desirable when the human is unable to do unsafe and unhealthy work. It gives assistance to the human because they never get tired and bored on repeated actions, have ability to do tasks in dangerous and uncomfortable situations and perform every task that unlike humans. Humanoid robots are utilized in assisting human activities because of their flexible and friendly appearance [11], [12]. However, it finds that control mechanisms are significantly less advanced. Most of the robots having fixed set of routine codes, their controlling mechanism perform inflexible reactions according to the situation. The learning systems of that robot was not based on psychological findings, so human feel difficulties to interpreting the obtained resulting knowledge. Cognitive architecture gives solution for that problem because their software systems provide support to develop human like cognition and set goals for general intelligence [13].

A cognitive architecture describes the fundamental structure of an intelligent system. It includes those aspects of cognitive systems that share different theoretical assumptions over time. Mostly of these theories belong to the skill acquisition, human memory, problem solving, and reasoning. These architectures based on effective and efficient construction of knowledge-based system that are developed using the programming languages and software environment [14]. Cognitive systems forecast future happening through selecting actions and learn from these events they perform and after that they modify successive expectations. Previously, the cognition was used as the symbol processing unit of the brain that concerns with rational planning and reasoning. Now these early approaches are changed and observe a strong relationship between the cognition, perception and action [15], [16]. The important role of cognitive architecture appears as the central goal of the artificial intelligence and cognitive science that support the similar abilities like human [17]. 
Humanoid robots can be beneficial from the strong cognitive abilities of these architectures provided. It is called a biological system that takes different decisions and behaves in the environment, learns from them and adapts how to react in new situation and provides solutions based on previous experience. The outstanding feature of the humanoid is the probability to communicate with it, to teach, to interact, and even to demonstrate. They can be used to predict the plans and effects regarding actions in order to accomplish the desire goals [19]. It is a challenging task to develop the connection of human with a robot and examines the effects of how systematically providing knowledge, intentions, and personality to the robot continuously over time. Many of the software are developed for humanoid robots that are control under Open Source paradigm, which shows numerous developers will be capable to modify these robots with powerful cognitive abilities. There are number of humanoid robot in worldwide, some recently developed robots such as NimbRo-OP [18], CASLHR [19], OpenCog [20], [21], and iCub [22], [23] having open source framework that have capabilities to interact with the environment and trying to achieve the required goals. They utilized their already defined pattern, stored memory, receive some information from the surrounding that are not enough for robot to work accurately and efficiently. These architectures have lack of learning and adapting ability, and exhibit limited behaviors. They take actions based on the pre-defined instructions that are given to them but they are unable to learn from previous attempt and update these actions for future use. They show only targeted or specific behaviors toward critical situation. Much of the progress has been done in the development of humanoid robots that are able to perform human-like behavior but still there are some deficiencies in the robots that need to overcome to perform like a real world. Humanoid robots must show behavior like the human being and having perfect intelligence, cognitive process, and perception used multiple behaviors according to the particular situation.

This paper demonstrates the consequences for the inactive method to cognition, phylogenetic pattern, create perception and perform the valid action, the significance of humanoid embodiment, reduce the empirical factual knowledge through the experience gets from the surrounding and their cognitive problems are determined based on user experience. In this regard, this research proposed a new architecture that has powerful cognitive ability for self-learning in humanoid robot with an interactive manner. A new proposed Innovative Cognitive Architecture for Humanoid Robot (ICAHR) having three modules: 1) Active Memory (AM); 2) Decision Processor (DP); 3) Sensor Listener (SL). The proposed architecture has ability to assist continuously for validating and adapting the real-world tasks, as it will demonstrate the attributes are very closer to the human behavior. In addition to this proposed structure, the sensor listener module will enable experimentation and evaluation of sensing capabilities, and emphasize their importance in the development of cognitive abilities. ICAHR architecture can perceive and feel like humans and has ability to learn based on its experience from previous attempt and modify its actions according to successive rate of its attempt to accomplish goals. The results show that the proposed architecture is suitable for the development of ICAHR architecture and performs much better than the existing humanoid robot architectures. ICAHR architecture shows real human like behavior having perfect cognitive process, perception and intelligence that engage in multiple behaviors according to the specific situation.

The remaining paper is organized as follows: Section II presents the materials and methods that explain the proposed Innovative Cognitive Architecture for Humanoid Robot (ICAHR). Section III includes the results and discussion in which explain the case studies between proposed ICAHR architecture and the conventional robots and Section IV includes the conclusion and future work of this research.

\section{MATERIALS AND METHODS}

This section explains the proposed Innovative Cognitive Architecture for Humanoid Robot (ICAHR) that is capable to work same as human cognition. Due to this similar cognition, it can be able to do more decision-making power in various situations, thinking, modify and respond actions based on previous experience. This architecture will try to produce intelligence same as the human by evaluating the internal operations and the architecture of the human brain. ICAHR architecture consist of three modules that describe the overall structure of humanoid robots of self-learning process. It contains autonomous philosophical memory like human which means that it can be able to think independently and show different attitudes towards same situation. Autonomous philosophical memory includes the AM and DP modules. Firstly, the AM module contains entity, entity relationship, pattern, action, and goals that have autonomous learning capabilities by actively explore and interact with the environment. AM module is capable to memorize new and old happening as well as memorize the social connections with the other people. Secondly, the DP module includes the perception, feeling processor, execution manager, and validator. This module is used for input-output, it processes the Mata data or information that is to perform more valid action to accomplish goals. Finally, the SL module that contains the active performer and action analyzer. It gets sense from external environment using action analyzer and for processing the observe information send it to the other modules. Based on processed information, the action performer conveys this information to the external environment according to the situations. ICAHR architecture are trying to train the learning behavior of humanoid robot to simulate the human brain activities, understand the planning approach, reasoning, judgment, awareness, representing in traditional artificial intelligence and show dynamic behavior of robot like a human in the surroundings. The selfmodification in proposed architecture depends on learning process and structure of the model due to that it is able to alter its model dynamics on the basis of experience, expand and increase its repositories of actions, and as a result modify new situation with more valid set of actions. 


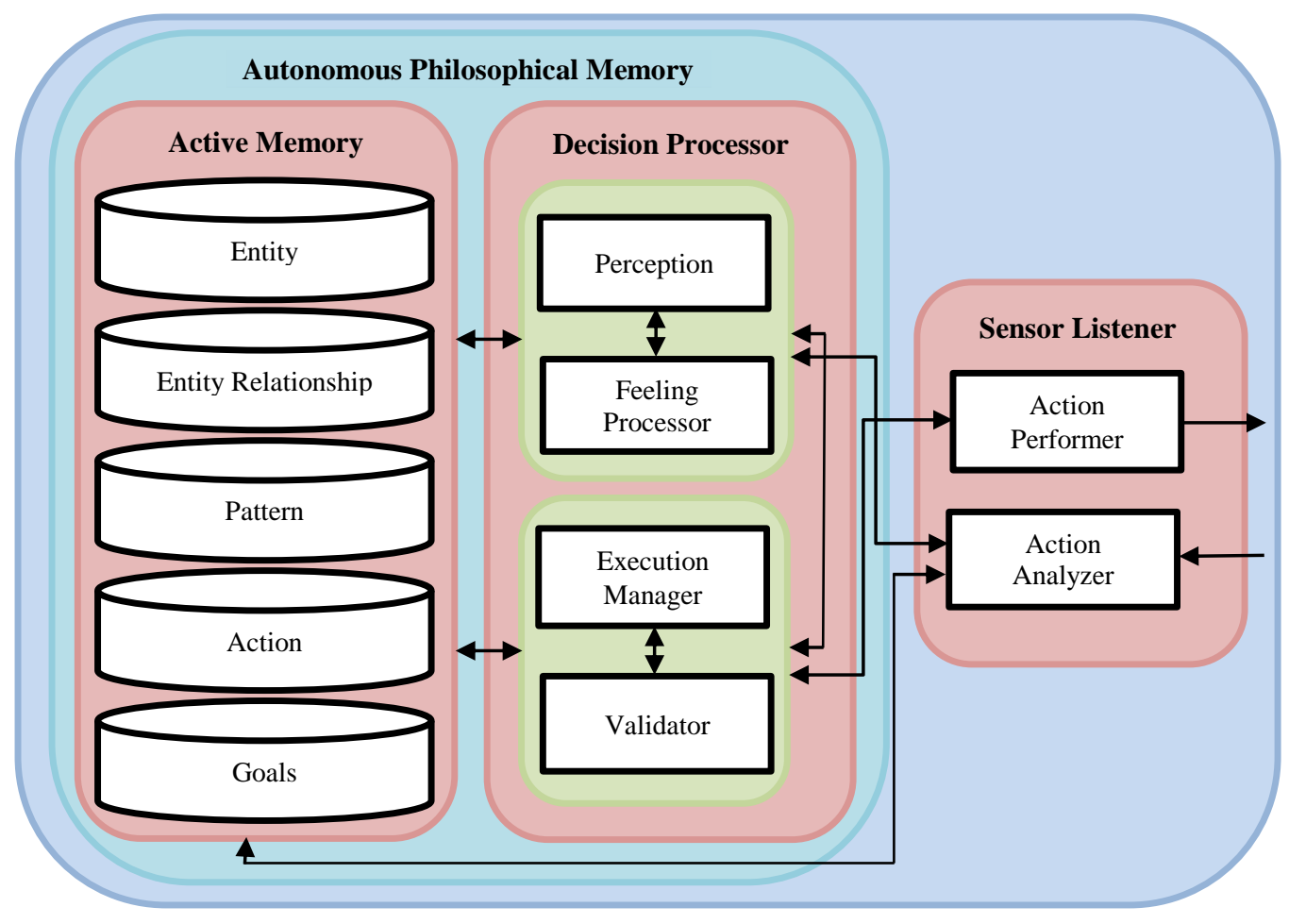

Fig. 1. Innovative Cognitive Architecture for Humanoid Robot (ICAHR).

Each module has individual relationship with the other module as explained in Fig. 1. Action analyzer get inputs from the environment and investigate the set of actions, make perception according to the situation and analyze the previously existence of similar actions in AM module. Based on the feelings and perception, it generates particular actions that fulfil the input commands for the purpose of achieving goals. The action performer is responsible to perform valid set of actions that are taken from DP module. More details of these modules are demonstrated as follows:

\section{A. Active Memory}

Active memory is used to memorize the set of actions and used to predict the effects of actions and takes the right decision for actions to accomplish the desire goals. The generation of information and reuse by contents in the system is facilitated through AM that can be stored permanently and retrieved same as the human brain. It is based on advance cognitive processing that is generally and particularly for learning. The main important goal of AM is employed as repositories for knowledge that is related to the interaction with the world and individual regarding series of activities. The repositories can be improve using autonomous learning process when the robot interact with the environment and improve their actions accordingly. Both memory and learning are the important aspects of cognition, in which the development of intelligent skills like deliberative reasoning, planning and self-regulation. ICAHR architecture used the operations of AM that update the memory's content include the entity, entity relationship, pattern, action and goals. When the robot interacts with the real world, it searches the element of perceptual memory that contain the current partial view of relevant surrounding entities. According to the observe entities, it makes the relationships between these entities and only the useful actions store in memory that exactly match to achieve a desire level of goals. All other entities are only beneficial for the specific moment. The observe entities are recognized like voice, face, object, obstacle and gesture are types of percept that are utilized to process actions, which is basically delivered into the AM. Every repository in AM has individual relationships with the DP module. The information will be store in AM autonomously based on the observation from environment. The knowledge of AM is used to facilitate prediction of future outcomes, imagination regarding new circumstances, and explanation of observed outcomes like the human.

When the robot observes all the real-world objects then these objects consider as entity. Every object that exists in entity repository appears to indicate distinction with the other entities or existence. ICAHR robot will store all these entities or objects in their memory and used to processes and modify the actions. In entity relationship repository, different entities relate to each other through a relationship and it define memory process systematically. The process is illustrated as components that are connected with each other by relationships, it expresses requirement and dependencies between them. Entity relationship is utilized in AM to figure out the entities, their attributes and relationship between the entities.

Pattern repositories are basically the arrangement of entities and their relationships that guide the robot about the angle of the movements and directions of its body parts or as a whole. It also observes the pattern and placement of all components (entities) in its surroundings. Finally, it plans valid scheme of its angle of movements on which follow to 
move its parts to achieve a successful action. ICAHR robot can be categorized into naive and learned pattern behavior. When the AM receives first time of stimuli of any entities and their relationships, it has no experience whether the proposed selection of pattern will succeed the goal or not. If the proposed pattern is succeeded, then it will store this successful pattern into memory for future use and if the attempt isn't successful, it will revise its pattern to accomplish the goals. Learned behavior of pattern appears when the AM find similar entities and their relationships again, its learned pattern for execution makes the previous attempt successful. AM will constantly update its patterns based on the experience and memorize only the successful attempt of patterns.

Action repository is generated by the combination of patterns. A step by step movement of body parts that makes the attempt successful will specify an overall action. However, it is necessary that all correct selection of patterns may lead to successful actions. Some cases of the angle and movement of robot lead to correct pattern but it performs action at the wrong target. So, it needs to perform the action again to achieve its right selection of targets. Due to that action can also be categorize into naive and learned behavior. For the first attempt of action, it has no experience regarding which action perform more better result and achieve its targets. Once the successful action performed with specific set of patterns, it will remember these actions in memory for future use. Based on experience, it shows learned behavior to perform action with better precision. Sometimes the learn repository provides considerable assistance to the robot by selecting the appropriate action to attempt. The ICAHR architecture for robot contains strong learning ability to focus on those potentially successful actions to achieve valid task like the human intelligence.

The combination of pattern may not ensure a correct action but the correct set of actions may lead towards the successful accomplishment of the desired goal. The robot will memorize all valid set of patterns and actions that makes the successful goal achievement and based on the achievement, it will update their repository. The proposed architecture emphasizes on the role of goals based on performance guidance. The ICAHR robot is able to compute goals according to its plans and retrieve them when it gets signal from working environment that it can be achievable.

\section{B. Decision processor}

Decision processor is used to process the information that is employed to perform action and to take attempt for the movement of robot towards the destination. This module includes the perception, feeling processor, execution manager and validator that are basically used for input and output to perform actions to achieve targets. When the robot get input from action analyzer, it will move to DP for further process. The robot figures out numerous perceptions that is used for the learning of action. Perceptions process continuously engages with different activities. It needs to know about the target and which object is presented before the movement of manipulator towards the engagement. The learning of robot is more manageable, if the robot perception is limited to the specific related task. ICAHR robot can figure out the feeling through facial or postural, vocal and emotional expression show a strong massage that share between the individuals in shared space. Robot feelings begin in sequence or with fluctuating time intensity like the human as naturally occurring emotions that often lead the stimuli to produce them. The important feature of feeling processor is used to coordinate the shifting behavior hierarchies, behavior response, control, promoting physiology support, interacting and establishing position related to other objects. Perception and feeling processor in DP that are mutually work together to perform collaborative task. Sometimes the robot observes the environment than show feelings based on the perception, in some cases the robot first feels something and make perception according to the situation and perform actions. Robot feelings are efficiently and effectively work with the human interaction, it means that the consideration of internal significance of the feeling processor is important for the robot performance. So, that the set of signals are prepare through the perceivable plans for the human-robot association. Perception and feeling processer play an important role as well as attention control, dialogue management, learning, communication elements and complex task planning.

The execution manager is basically responsible to determine the behavior of ICAHR robot throughout the interaction with the real world. This unit is considered as the fundamental component of decision maker that has capabilities to handle the complex environment. Execution manager will formulate a strategy based on the feeling and processor to perform actions for accomplishing goals and this formulated scheme will further move to the validator. Finally, the validator is the superior task to take the final decision and re-confirm the actions. The scheme that is taken from execution manager contains the sequence of actions in which every unit shows the different environmental intentions that contains one correct action for each unit. The correct selection of action will give positive feedback that enhance the reward function and it learns from right selection of commands. If the validator didn't accept the scheme than it will return to the execution manager for improving actions. Due to that the execution manager is more cautious for right selection of actions and strongly motivated to examine multiple times before send for the final decisions. Moreover, validator contains the set of queries regarding actions in the sort of verification question same as the human brain that can be ejaculated in some situation when it commands additional information.

The overview of the working of DP module can be defined as the processing of actions to achieve goals. Perception and feeling processor are basically a two-way process. Either the robot will develop a perception first and will show feelings about the environment and its prospect goals, or it may feel anything first and afterward it will make a perception. The expression of the perception and feeling will be represented in the form of gestures on the facial part of the humanoid robot. Execution manager will formulate a scheme based on the feelings and perception determined to perform an action for the accomplishment of goals. This formulated scheme passes the execution check in validation unit, then it will be implemented, but if the validation check rejects the scheme will be sent back for updating action to the execution 
manager. This scheme will only implement if the validation check passes the execution manager's proposed scheme.

\section{Sensor Listener}

Sensor listener module basically take sense from external world using action analyzer and sent these commands to the AM and DP module for processing. Similarly, it conveys the set of processed commands using action performer that taken from AM and DP to the external environment. Sensors are utilized for extracting appropriate views of the module of engagement. Based on the cognitive approach, this module is responsible to control the incoming and outgoing actions. Action performer takes commands from other modules to perform a set of actions in the form of interaction and communication with the real world and get feedback for further processing. SL module allows the robot to coordinate using the movement of eyes and hands for grasping and manipulating the objects having reasonable size and appearance, sit up, crawling with the arms and legs. It will allow robot to interact and explore with the world, not only through the manipulation of objects but also by locomotion. Moreover, the SL module of the robot will enable the evaluation and testing of the sensing abilities and display their significant role in the development of cognitive abilities.

The overall summary of proposed ICAHR architecture is said to be a complete framework of cognitive architecture for humanoid robots. This research is trying to develop a cognitive architecture that has broadly same as human intelligence as well as powerful self-controllable and sensible motivational system than human. ICAHR robot consist of strong cognitive abilities like the human memory and has better abilities to face multiple challenges when interacting with the surrounding. Robots are engaged with the real world by sharing activities during interaction in which every action of robot is influence with the actions of real world that results in some mutual constructed patterns of shared behavior. It has capabilities of self-learning which is based on feelings and perceptions as well as learning from experience. It is capable to modify actions and performs more valid actions when the action analyzer give command to take attempt. Humanoid robot stores all successful actions in the memory. Also, when similar situation occurs in future than their self-learning behavior motives to performs more effectively and efficiently based on experience. It has thinking capabilities to plan new strategies for right decision of actions to achieve targets. The cognitive abilities of ICAHR robot includes creativity, thinking, awareness, imagination, feelings, perception, decision making, reasoning, desires, ideas and self-examining. The proposed architecture develops knowledge through interaction with the real world and it captures the invariance and consistency that appears from the dynamic selforganization in the aspect of environmental connection. The capability of cognition and artificial intelligence make this architecture same as the human brain.

The comparison of conventional robots and proposed architecture are illustrated in Table 1. The issues with the conventional robots are deficiency of effective information processing having inadequate capabilities to learning from environment and perform limited tasks that already programmed. As compared with the conventional humanoid robots, proposed architecture are strong abilities of learning and thinking that display versatile behavior. It performs valid actions consisting of cognitive abilities and artificial intelligence. The comparison table explains different situations which revealed that the proposed architecture is more efficient and flexible than the conventional robots.

TABLE I. COMPARISON OF CONVENTIONAL ROBOTS AND ICAHR ARCHITECTURE

\begin{tabular}{|l|l|}
\hline Conventional robots & ICAHR architecture \\
\hline $\begin{array}{l}\text { Conventional robots consist on } \\
\text { memory that stores only already } \\
\text { programmed instructions about } \\
\text { the environment. }\end{array}$ & $\begin{array}{l}\text { Proposed ICAHR architecture } \\
\text { contain active memory that is } \\
\text { capable to store programmed } \\
\text { instructions as well as empirical } \\
\text { information regarding environment. }\end{array}$ \\
\hline $\begin{array}{l}\text { They are unable to distinguish } \\
\text { between relationships of } \\
\text { different entities. }\end{array}$ & $\begin{array}{l}\text { It can be identifying the relationship } \\
\text { between different entities and make } \\
\text { patterns for performing actions } \\
\text { according to the entity relationship. }\end{array}$ \\
\hline $\begin{array}{l}\text { They show insufficient mobility } \\
\text { that already programmed. }\end{array}$ & $\begin{array}{l}\text { It shows efficient mobility based on } \\
\text { the situation's experience. }\end{array}$ \\
\hline $\begin{array}{l}\text { They present limited reaction } \\
\text { according to any stimuli. }\end{array}$ & $\begin{array}{l}\text { It is capable to display flexible } \\
\text { reactions based on any stimuli. }\end{array}$ \\
\hline $\begin{array}{l}\text { If same situation happened, they } \\
\text { perform similar actions } \\
\text { repeatedly. }\end{array}$ & $\begin{array}{l}\text { It has capabilities to show different } \\
\text { reactions towards the similar } \\
\text { circumstances based on learned } \\
\text { behavior. }\end{array}$ \\
\hline $\begin{array}{l}\text { They are unable to store } \\
\text { instructions temporarily. }\end{array}$ & $\begin{array}{l}\text { It can be store instructions } \\
\text { temporarily as well as permanently. }\end{array}$ \\
\hline $\begin{array}{l}\text { They are unable to recognize } \\
\text { feelings and emotions. }\end{array}$ & $\begin{array}{l}\text { It is capable to recognize the } \\
\text { feelings by using feelings processor } \\
\text { in DP module. }\end{array}$ \\
\hline $\begin{array}{l}\text { They are unable to learn actions } \\
\text { from real world. }\end{array}$ & $\begin{array}{l}\text { It can be able to learn actions from } \\
\text { environment and store set of actions } \\
\text { in the memory. }\end{array}$ \\
\hline
\end{tabular}

\section{RESUlt AND DISCUSSION}

To evaluate the cognitive abilities of self-learning of ICAHR robot by using the case studies and apply these case studies into conventional robots and proposed ICAHR architecture is demonstrated on it. Firstly, considering the case studies that robot directs the people on the way to IT lecture room. Secondly, the robot shares its experience to the peoples about assisting people on the way to the target location. More details of these case studies are demonstrated as follows:

\section{A. Case A: Robot Directs the People on the Way to IT Lecture Room}

This case study can be divided into smaller activities and each activity is implemented in the most effectively and efficiently manner such as the self-autonomous working of the robot toward the target, step by step navigation of the robot for the direction of IT lecture room, finds the specific area of IT lecture room, reached the lecture room and finish its task, after that it come back to the front of the building. Navigation of robot is done with different sensors when its functioning toward the target. ICAHR robot need assistance with different sensors to properly navigate by floor plan without disturbing 
and touching any surrounding objects. Sensors can locate the target position for the robot, so it can assist people toward specified location. Firstly, applying this case study into the conventional robots and then implement it into the proposed ICAHR architecture.

1) OpenCog: When the robot gets the commands about assisting the people those who want to go to IT lecture room and help them toward the right location, it store these instruction signals into the memory called atom space. These atom spaces are linked with each other and communicate with mind agent. Mind agent performs some cognitive actions because it has no capability to do the variety of decisions towards the targets and to learn the new behaviors due to its less intelligence. It performed actions through the mind agents that to locate the specified area of room.

2) $i C u b$ : When the robot gets instructions by sensing that someone want to go to the IT lecture room and request the robot to help him on the way to the right location. iCub robot will sense these signals by agents and save these instructions into the episodic memory. It will verify that these instructions already exist in episodic memory or not, if robot identify that pre-guided instructions then it will executes its effective state and passes these instruction signals to the selection of actions that navigate of robot toward the faculty building and search the already programmed location and reached the specified area of lecture room.

3) NimbRo: When this robot gets instructions to direct the people on the way to IT lecture room, it is not capable to perform these actions because it is only develop for basic soccer skills and has specifically programmed to play only football game. If this robot is utilized for the purpose to assist peoples toward lecture room, it needs to be programmed to perform these kinds of actions that how to sense the assistance instructions from the people and perform specified actions.

4) Proposed CASLHR: When ICAHR robot gets the commands to assist people on the way to IT lecture room then first of all, it will sense the lecture room area through the action analyzer operation in SL module and send these commands for processing to the $\mathrm{AC}$ and $\mathrm{DP}$ module. The process of each module is explained in following steps:

Step 1: Active memory module includes some operations that the ICAHR robot is initially containing a set of actions to accomplish ultimate goals and after that successful actions are utilized to store into the memory for future use similar as human intelligence. Entity repository in AM includes as follows: building, corridor, offices, stairs, peoples, lecture rooms, doors, chairs and tables. Similarly, the entity relationship repository contains the following set of relations with the entities: the building has relation with offices and stairs, offices are used by the staff or people, lecture rooms have a door and it contain chairs and tables, other things have direct or indirect relation with building offices, and people, lecture room.

The ICAHR robot will start moving after getting instructions from people. The movement of the robot towards the building is done through information provided by sensors, find the targeted location after passing from stair and offices. Robot detects the lecture room title on the door by using sensor. The robot performs several actions like starts automatically when getting instructions from people, moving toward the destination by sensing lecture room titles on the door, after assisting the people on targeted location, it will return in front of the building. The ultimate goal of humanoid robot is to find the targeted lecture room and performs necessary actions that try to save time, tiredness, stress and give motivation for studies.

Step 2: This step discusses how DP module work with robot when getting call for assistance from surroundings. Firstly, the feeling processor takes instruction signals from people and automatically starts the robot. It gives the sense to the robot what he will do after listening the voice of peoples and change his behavior for specific action. Perception operation makes sense to the robot that someone call for assistance and the execution manager directs him for the movement toward target. The validator is to re-confirm the execution manager action and humanoid robot started his movement when the validator guides the SL to proceed this action. Secondly, feeling processor detects the lecture room title on the door with the help of sensor. Humanoid robot makes the perception that still targeted goal is not achieved. The execution manager gives the directions to the robot to keep moving until reached at the target. The validator operation step by step verified the execution manager actions and guide the robot to keep moving towards the target when the validator send signal to the SL to proceed these actions. Finally, in feeling processor the sensor continues to detect the lecture room door by door and the robot makes the perception that he reached to the targeted area of the building. The execution manager wants to stop the robot in front of the IT lecture room and makes them decision. The validator operation validates the actions of execution manager and robot asks the people this is your destination when the validator sends instructions to the SL module.

Step 3: The action performer in SL module gets a set of commands from validators that to perform these actions. This module is used to sense the set of commands using action analyser and after processes these commands from AM and DP to get a set of more valid actions for executing specified tasks such as listen the people voice, robot start its movement, continue its movement until it sense to reach the specified location.

\section{B. Case B: Robot Share its Experience with the Peoples}

When the robot meets with the real world, communicate with the peoples about how he can gain experience on the way to target location of IT lecture room and explain the hurdles that he faces on the way. Similarly, applying this case study into the conventional robots gives implementation on the proposed ICAHR architecture.

1) OpenCog: When the OpenCog robot receives set of instruction from the peoples to sharing his experience how to direct people on the way to IT lecture room, then it stores these set of instructions into the memory. Atom spaces are 
utilized as memory in the OpenCog robot which are connected with each other that ensure the set of instructions already exist in atom space or not and after it communicate with the mind agent. The mind agent executes some cognitive process due to less intelligent. The mind agent performs the set of actions that share experiences how he finds the building that contain IT lecture room and reached the specified lecture room.

2) iCub: When the iCub robot gets commands from the peoples to share his experience that how you accomplish your specified target to reached at the destination lecture room. Robot will sense these set of commands by agent and store into the episodic memory. It will determine that these commands already happened in episodic memory or not. If the robot find out these pre-programmed commands exist then it will run its effective state process. Further, it pass these commands to execute actions that share main steps how effectively identify the building, how to sense the specified lecture room in the building.

3) NimbRo: When the NimbRo robot gets instructions from the peoples to share its experience that how he successfully reached the destination. This robot is not capable to perform any actions regarding sensing the target location in the building because it have limited programmed that is only used for the football game. For the purpose of searching specified location in building, this robot needs to be programmed for executing location searching task then it will able to share any experience.

4) Proposed ICAHR: When ICAHR robot communicates with the peoples in the real world, then the sensor gets set of commands about how he gain experience on the way to assisting people toward the specified lecture room. Robot shares its experience that how fast he sense the location of lecture room in the building, how efficiently and effectively reached the specified target in the building, how many obstacle faces toward the IT lecture room. So, the ICAHR robot examined the working pattern of human-like intelligence and it shows a clear example of experience based learning. At first attempt, the robot was naive to the set of patterns and actions about the location search in the building, whether the goal is achievable or not. Robot arbitrarily moving on the way to the lecture room and one by one observe all the room titles until the destination location. Robot initially set the patterns and actions were constantly revised to make a successful attempt. After finalizing the perception and feeling of the robot, it finally makes the strategy to continue moving on the particular area. This strategy will be validate by the validator after examining all parameters and then this strategy implemented by action performer in SL module. Validator forward only those commands that ensures successful attempt when executed otherwise it will return to the execution manager for modification in their decision. If the same situation occurs in second time, a learned behavior of robot was observed based on the past experience. Thus, the previous experience of the humanoid robot brings the more valid actions and having conscious intellectual capability to reduce empirical factual knowledge. The robot strategy for attempt was verified by the validator and it tries to improve its actions and behaviors constantly that provide a similarity to a humanlike behavior. It senses the people instruction in the real world and responds it accordingly. Many time experiences in particular situation makes the overall output as a successful attempt.

TABLE II. Summary of CASE StUdies BETWEen CONVENTIONAL RoBots ANd PROPOSED ICAHR ARChiteCtURE

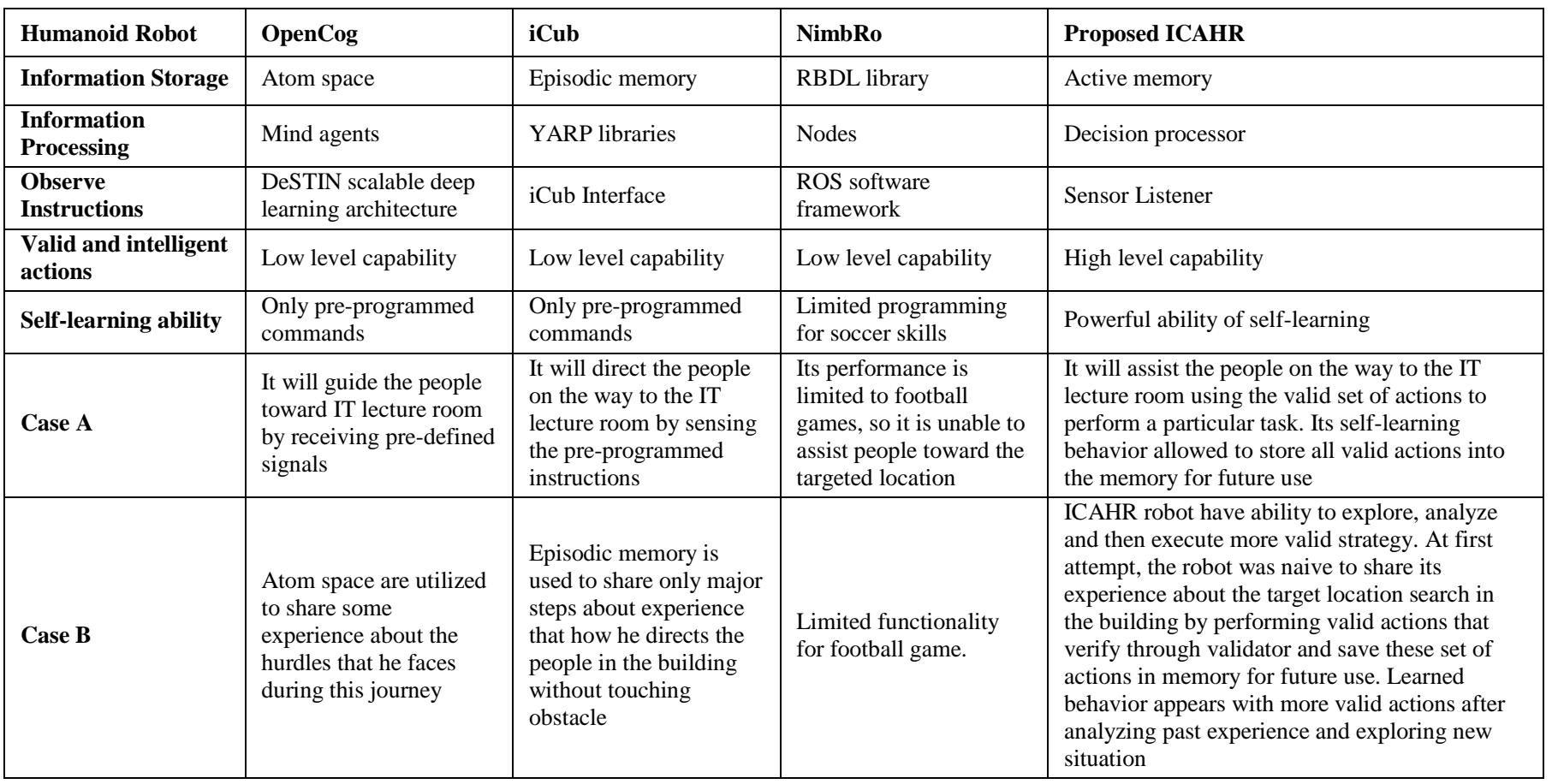


The results of above discussed case studies show that the conventional robot take actions based on the pre-programmed commands given to them and display lack of learning behavior. They used stored memory to get some instructions from the environment that is not enough to work accurately and efficiently. The summary of these case studies are mentioned in Table 2. The proposed ICAHR architecture overcome the deficiencies of conventional robots in much better way same as the human.

\section{CONCLUSION AND FUTURE WORK}

An approach for a cognitive architecture for an intelligent humanoid robotic system has been presented in this paper. The purpose of this research is to create a humanoid robot that can be capable of understanding the environment by communicate using gestures and simple expressions. This intelligence can achieve through strong manipulation that is based on exploration, imitation and social interaction. In this paper, we are presenting an innovative architecture to automate a robot which contains rich cognitive ability and their behaviour will be very closely related to the human. Prediction is involving in every action and each action will change the perceptual world to some extent. Similarly, every span of perception is basically associated or linked with an action. The ICAHR robot is compared with conventional humanoid robots, proposed architecture significantly improves the learning behaviour of robot from experience. It provides more effective and valid decisions in the real world and its features are almost same as a human. Validation check in DP module that can precisely validate the strategies and implemented actions more specifically for the successful achievement of goals. The key characteristic of ICAHR robot is to develop perception and feeling and to display a learned behaviour after validating actions. AM is capable to store temporary and permanent set of command and most important feature that it constantly learns from its experience and based on experience, it can continually update its actions in the AM. So, it gives rise to an ICAHR architecture is innovative approach to experience based learning. The future work of this research is to develop and programmed the knowledge-based learning system for the ICAHR architecture to make its functionality closer to the human cognition. If we upgrade the databases of AM and DP module then it will increase the learning ability and store information more accurately and systematically that can reduce the chances to unsuccessful attempt. This research can be further analysed to explore on emergent embodied systems which can develop strong cognitive skills because it will perform the actions in the real world and figure out the strong consequences clearly to adopt this stance.

\section{REFERENCES}

[1] D. Vernon, C. Hofsten, and L. Fadiga, A roadmap for cognitive development in Humanoid Robots, Springer-Verlag Berlin Heidelberg, 2011.

[2] L. Montesano, M. Lopes, A. Bernardino, and J. Santos-Victor, "Learning object affordances: from Sensory-Motor coordination to imitation," IEEE Trans. Robot., vol. 24, no. 1, pp. 15-26, 2008.

[3] T. Asfour, K. Yokoi, C. . G. Lee, and J. Kuffner, "Humanoid Robotics," IEEE Robot. Autom. Mag., pp. 108-118, 2012.
[4] T. Asfour, J. Schill, H. Peters, C. Klas, B. Jens, C. Sander, S. Schulz, A. Kargov, T. Werner, and V. Bartenbach, "ARMAR-4 : A 63 DOF torque controlled Humanoid Robot," IEEE-RAS Int. Conf. Humanoid Robot., pp. 390-396, 2013.

[5] J. S. Albus and A. J. Barbera, "RCS: A cognitive architecture for intelligent Multi-Agent systems," Annu. Rev. Control, vol. 29, no. 1, pp. 87-99, 2005.

[6] M. D. Byrne, "Cognitive architectures in HCI: present work and future directions," in Proc. 11th Int. Conf. Hum. Comput. Interact., 2005.

[7] W. Duch, R. Oentaryo, and M. Pasquier, "Cognitive Architectures: Where do we go from here?," Front. Artif. Intell. Appl., vol. 171, pp. 122-136, 2008.

[8] M. Frank and N. Franklin, Computational Cognitive Neuroscience, Wiki Book, 1st Edition, 2013.

[9] C. Green and J. E. Hummel, "Relational perception and cognition: Implications for Cognitive Architecture and the perceptual-cognitive interface," Psychol. Learn. Motiv. - Adv. Res. Theory, vol. 44, pp. 201226, 2003.

[10] Y. Wang, "On Cognitive Computing,” Int. J. Softw. Sci. Comput. Intell., vol. 1, no. 3, pp. 1-15, 2009.

[11] R. S. Lakshmi, "Renovating Robots," Int. J. Emerg. Technol. Eng. Res., vol. 3, no. 2, pp. 70-75, 2015.

[12] J. Pierezan, R. Zanetti, L. Weihmann, and G. Reynoso-meza, "Static force capability optimization of humanoids robots based on modified self-adaptive differential evolution," Comput. Oper. Res., pp. 1-11, 2016.

[13] K. Kim, D. Choi, J. Y. Lee, J. M. Park, and B. J. You, "Controlling a humanoid robot in home environment with a cognitive architecture," in Proc. IEEE Int. Conf. Robot. Biomimetics, pp. 1754-1759, 2011.

[14] R. Sun, P. Langley, J. E. Laird, and S. Rogers, "Cognitive architectures: Research issues and challenges," Cogn. Syst. Res., vol. 10, no. 2, pp. 141-160, 2009.

[15] J. R. Anderson, D. Bothell, M. Byrne, S. Douglass, C. Lebiere, and Y. Qin, "An integrated theory of the mind," Psychol. Rev., vol. 111, no. 4, pp. 1036-1060, 2004.

[16] P. Langley, "An adaptive architecture for physical agents," in Proc. IEEE/WIC/ACM Int. Conf. web Intell., pp. 18-25, 2005.

[17] P. Langley, "Intelligent behavior in humans and machines," Am. Assoc. Artif. Intell., pp. 3-12, 2006.

[18] M. Schwarz, J. Pastrana, P. Allgeuer, M. Schreiber, S. Schueller, M. Missura, and S. Behnke, "Humanoid teenSize open platform NimbRoOP,” Lect. Notes Comput. Sci. Rob. 2013 Robot World Cup XVII, vol. 8371 LNAI, pp. 568-575, 2013.

[19] Mushtaq, M. F., Khan, D. M., Akram, U., Ullah, S., \& Tariq, A., "A cognitive architecture for self learning in Humanoid Robots," Int. J. of Comp. Sci. and Net. Sec., vol. 17, no. 5, pp. 26-36, 2017.

[20] B. Goertzel, C. Pennachin, and N. Geisweiller, The CogPrime Architecture for Integrative, Embodied AGI, 2014.

[21] D. Hart and B. Goertzel, "OpenCog: A software framework for integrative artificial general intelligence," Front. Artif. Intell. Appl., vol. 171, no. 1, pp. 468-472, 2008.

[22] G. Metta, L. Natale, F. Nori, G. Sandini, D. Vernon, L. Fadiga, C. von Hofsten, K. Rosander, M. Lopes, J. Santos-Victor, A. Bernardino, and L. Montesano, "The iCub humanoid robot: An open-systems platform for research in cognitive development," Neural Networks, vol. 23, no. 8-9, pp. 1125-1134, 2010.

[23] N. G. Tsagarakis, G. Metta, G. Sandini, D. Vernon, R. Beira, F. Becchi, L. Righetti, J. Santos-Victor, a. J. Ijspeert, M. C. Carrozza, and D. G. Caldwell, "iCub: The design and realization of an open humanoid platform for cognitive and neuroscience research," Adv. Robot., vol. 21, no. 10, pp. 1151-1175, 2007. 